TITLE:

\title{
Ferromagnetism in sputtered manganese-doped indium tin oxide films with high conductivity and transparency
}

AUTHOR(S):

Nakamura, T; Tanabe, K; Tsureishi, K; Tachibana, K

\section{CITATION:}

Nakamura, $T$...[et al]. Ferromagnetism in sputtered manganese-doped indium tin oxide films with high conductivity and transparency. JOURNAL OF APPLIED PHYSICS 2007, 101(9): 09H105.

ISSUE DATE:

2007-05-01

URL:

http://hdl.handle.net/2433/50175

RIGHT:

Copyright 2007 American Institute of Physics. This article may be downloaded for personal use only. Any other use requires prior permission of the author and the American Institute of Physics. 


\title{
Ferromagnetism in sputtered manganese-doped indium tin oxide films with high conductivity and transparency
}

\author{
Toshihiro Nakamura, ${ }^{a}$ Kohei Tanabe, Kazuki Tsureishi, and Kunihide Tachibana \\ Department of Electronic Science and Engineering, Kyoto University, Kyotodaigaku-Katsura, Nishikyo-ku, \\ Kyoto 615-8510, Japan
}

(Presented on 11 January 2007; received 31 October 2006; accepted 2 December 2006; published online 30 March 2007)

\begin{abstract}
Mn-doped indium-tin oxide (ITO) films were deposited on glass substrates using radio-frequency magnetron sputtering. The magnetization as a function of magnetic field showed hysteretic behavior at room temperature. According to the temperature dependence of the magnetization, the Curie temperature is higher than $400 \mathrm{~K}$. Anomalous Hall effect was observed at low temperatures. Ferromagnetic thin films of Mn-doped ITO exhibited low electrical resistivity of $3.9 \times 10^{-4} \Omega \mathrm{cm}$ and high optical transmittance between $75 \%$ and $90 \%$ in the visible region. (C) 2007 American Institute of Physics. [DOI: 10.1063/1.2710324]
\end{abstract}

\section{INTRODUCTION}

Diluted magnetic semiconductors (DMSs) have gained much attention because of their potential applications in spin electronics. ${ }^{1,2}$ High-temperature ferromagnetism has been observed in oxide-based DMS. ${ }^{3-21}$ Indium tin oxide (ITO) is one of the most commonly used transparent conducting materials in various optoelectronic devices such as displays and solar cells because of its metal-like electrical conductivity and excellent optical transparency. Integrating ITO into magneto-optoelectronic devices is straightforward, since there are several devices using its thin films in the market. Recently, high-temperature ferromagnetism has been reported in some magnetic impurity doped ITO-based systems. $^{3-5}$ In this work, we prepared Mn-doped and undoped ITO films on glass substrates by radio-frequency (rf) magnetron sputtering, and investigated not only the magnetic property of Mn-doped ITO films but also the effect of the manganese doping on the film quality by comparing the electrical and optical properties between Mn-doped and undoped ITO films. In addition to high-temperature ferromagnetism, anomalous Hall effect was observed in the Mn-doped ITO films. Ferromagnetic thin films of Mn-doped ITO with low electrical resistivity of the order of $10^{-4} \Omega \mathrm{cm}$ and high optical transmittance were obtained.

\section{EXPERIMENT}

Thin films were deposited on glass substrates from a Mn-doped ITO target using rf magnetron sputtering. The target with the atomic ratio of $\mathrm{In}: \mathrm{Sn}: \mathrm{Mn}=17: 2: 1$ was used. After the chamber was evacuated to a high vacuum of about $1 \times 10^{-6}$ Torr, working gas of argon was introduced. Before the deposition, the target was presputtered for $30 \mathrm{~min}$ to obtain a clean target surface. The process pressure was controlled at 13 mTorr during the sputter deposition. The $\mathrm{rf}$ power was $200 \mathrm{~W}$, which led to a deposition rate of about

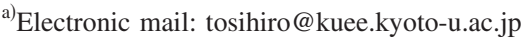

$15 \mathrm{~nm} / \mathrm{min}$. The deposition temperature was $200{ }^{\circ} \mathrm{C}$. The deposited films were annealed in $\mathrm{N}_{2}$ at $300{ }^{\circ} \mathrm{C}$ for the improvement of the electrical properties.

The atomic composition of the deposited films was evaluated by x-ray photoelectron spectroscopy (XPS) after etching the film surface. The film thickness was obtained by cross-sectional scanning electron microscopy. All films were about $300 \mathrm{~nm}$ thick. Transport properties (resistivity, carrier density, and mobility) of the deposited films were measured by Hall effect measurements using van der Pauw geometry. Optical transmission spectra in the wavelength region of 300-800 nm were acquired using a UV-visible spectrophotometer. Magnetic properties were characterized by a superconducting quantum interference device (SQUID) magnetometer. A six-point Hall bar geometry was used to obtain anomalous Hall effect signals.

\section{RESULTS AND DISCUSSION}

In order to confirm the incorporation of manganese atoms in the deposited film, XPS measurements were carried out. Figure 1 shows a typical XPS spectrum of Mn-doped

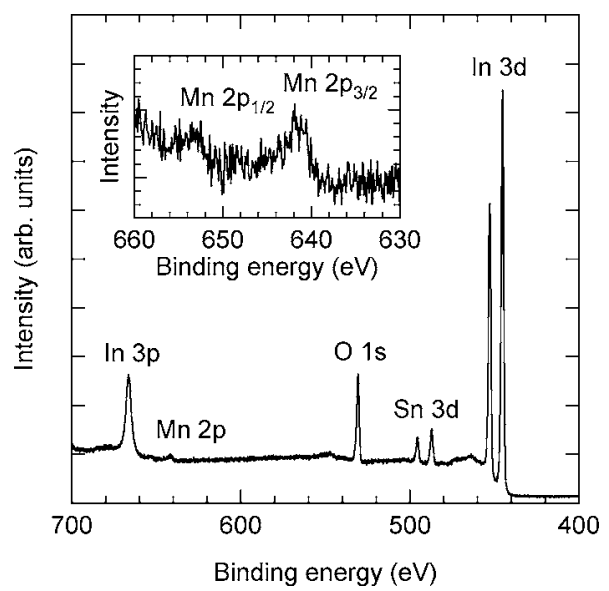

FIG. 1. XPS spectrum of the deposited Mn-doped ITO film. The magnification of the Mn $2 p$ peaks is shown in the inset. 


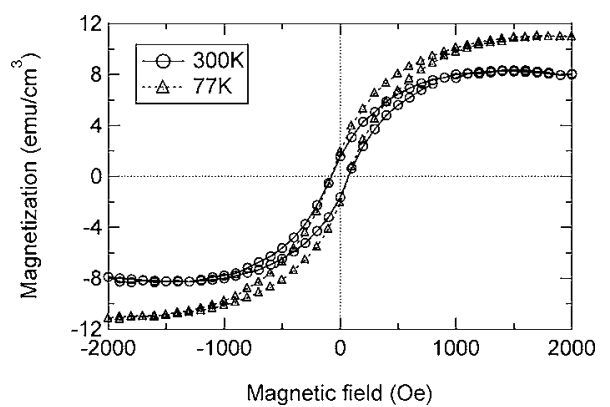

FIG. 2. Magnetization vs applied magnetic field curves measured at 77 and $300 \mathrm{~K}$ for the Mn-doped ITO film.

ITO films. As shown in the inset, Mn $2 p$ peaks around $640 \mathrm{eV}$ were observed in the XPS spectra of Mn-doped ITO films. The relative atomic concentration of each element was calculated according to the peak areas and relative atomic sensitivity factors. The $\mathrm{Mn}$ atomic concentration in the $\mathrm{Mn}$ doped film was evaluated to be $3 \%$ using the Mn $2 p$ signal.

The magnetization versus applied magnetic field curves measured at 77 and $300 \mathrm{~K}$ for the Mn-doped ITO film are shown in Fig. 2. The magnetic field was applied parallel to the film plane. A well-defined hysteresis loop with a coercive force of about $80 \mathrm{Oe}$ was observed at $300 \mathrm{~K}$, signifying room temperature ferromagnetism. Figure 3 shows the temperature dependence of the magnetization for the Mn-doped ITO film measured from 5 to $400 \mathrm{~K}$ using SQUID. The magnetization data were taken in an applied field of $1000 \mathrm{Oe}$ parallel to the film plane. The Curie temperature is estimated to be higher than $400 \mathrm{~K}$. Figure 4 shows a Hall resistivity versus applied magnetic field plot measured at $5 \mathrm{~K}$. The dotted line denotes the ordinary Hall effect component. The figure clearly indicates the anomalous Hall effect at low magnetic field, corresponding to the nonlinear part of the curve. The evidence of anomalous Hall effect suggests that the charge carrier interacts with the local magnetism in the $\mathrm{Mn}$ doped ITO films.

Undoped ITO films were grown at the same deposition conditions to compare the electrical and optical properties between Mn-doped and undoped ITO films. Transport properties (resistivity, carrier density, and mobility) of Mn-doped and undoped ITO films were measured by Hall effect measurements using van der Pauw geometry. The manganese doping increased the resistivity from $2.6 \times 10^{-4}$ to 4.3 $\times 10^{-4} \Omega \mathrm{cm}$. The increase of the resistivity was mainly due to the decrease of the mobility. In order to improve the elec-

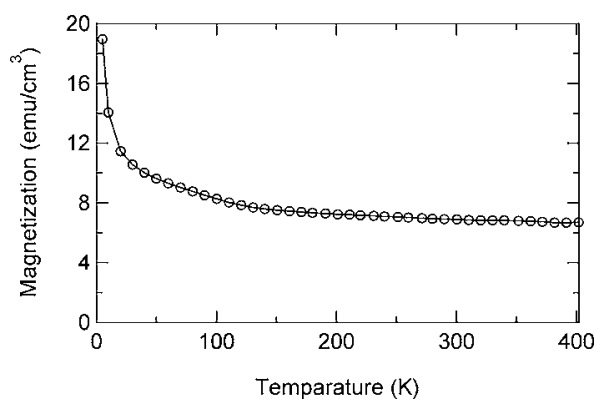

FIG. 3. Temperature dependence of magnetization for the Mn-doped ITO film under applied magnetic field of 1000 Oe.

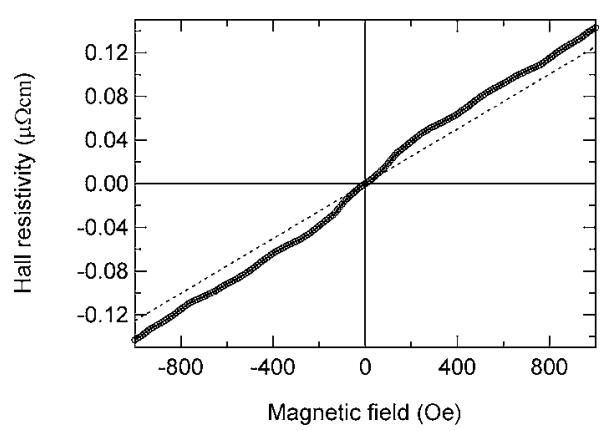

FIG. 4. Hall resistivity vs applied magnetic field plot measured at $5 \mathrm{~K}$ for the Mn-doped ITO film. The dotted line denotes the ordinary Hall effect component.

trical properties, the deposited films were annealed in $\mathrm{N}_{2}$ at $300{ }^{\circ} \mathrm{C}$. This annealing treatment increased slightly both the mobility and carrier density of the films. As a result, ferromagnetic Mn-doped ITO films with low electrical resistivity of $3.9 \times 10^{-4} \Omega \mathrm{cm}$ were obtained. The carrier density and mobility of the present Mn-doped ITO film were 1.4 $\times 10^{21} \mathrm{~cm}^{-3}$ and $13 \mathrm{~cm}^{2} \mathrm{~V}^{-1} \mathrm{~s}^{-1}$, respectively.

The optical transmission spectra for Mn-doped and undoped ITO films are shown in Fig. 5. The transmittance exhibits interference in the visible range. The average transmittance of the Mn-doped ITO film ranges from $75 \%$ to $90 \%$. The Mn-doped ITO film is optically highly transparent, although the Mn-doped ITO film has an absorption edge at a higher wavelength than the undoped ITO film.

In summary, Mn-doped ITO films were deposited on glass substrates by rf magnetron sputtering. The magnetic measurements indicated that the Mn-doped ITO films are ferromagnetic above room temperature. The anomalous Hall effect was observed in magnetotransport measurements, which suggests that the ferromagnetism in the deposited films is intrinsic. Ferromagnetic thin films of Mn-doped ITO with low electrical resistivity of the order of $10^{-4} \Omega \mathrm{cm}$ and high optical transmittance were obtained successfully. The coexistence of ferromagnetism, high electrical conductivity, and high visible transparency in the Mn-doped ITO films is expected to be a desirable trait for spin electronics devices.

\section{ACKNOWLEDGMENTS}

We gratefully acknowledge Professor M. Suzuki and Dr. T. Kawakami of the Department of Electronic Science and Engineering at Kyoto University for their help in the magnetic measurements. We would like to thank Kyoto Univer-

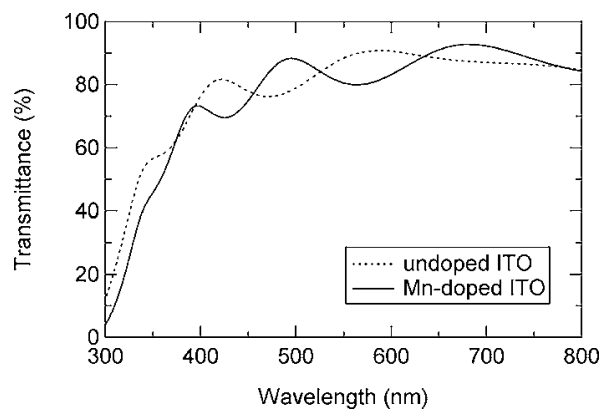

FIG. 5. Optical transmission spectra for Mn-doped and undoped ITO films. 
sity Venture Business Laboratory (KU-VBL) for the support. This work was partly supported by Kyoto Nanotechnology Cluster and Nanotechnology Support Project of the Ministry of Education, Culture, Sports, Science and Technology (MEXT), Japan.

${ }^{1}$ H. Ohno, Science 281, 951 (1998).

${ }^{2}$ S. A. Wolf, D. D. Awschalom, R. A. Buhrman, J. M. Daughton, S. von Molnar, M. L. Roukes, A. Y. Chtchelkanova, and D. M. Treger, Science 294, 1488 (2001)

${ }^{3}$ J. Philip, N. Theodoropoulou, G. Berera, J. S. Moodera, and B. Satpati, Appl. Phys. Lett. 85, 777 (2004).

${ }^{4}$ H. S. Kim, S. H. Ji, H. Kim, S.-K. Hong, D. Kim, Y. E. Ihm, and W. K. Choo, Solid State Commun. 137, 41 (2006).

${ }^{5}$ G. Peleckis, X. Wang, and S. X. Dou, Appl. Phys. Lett. 89, 022501 (2006).

${ }^{6}$ J. M. D. Coey, M. Venkatesan, and C. B. Fitzgerald, Nat. Mater. 4, 173 (2005).

${ }^{7}$ Y. Matsumoto et al., Science 291, 854 (2001).
${ }^{8}$ K. Ueda, H. Tabata, and T. Kawai, Appl. Phys. Lett. 79, 988 (2001).

${ }^{9}$ P. Sharma et al., Nat. Mater. 2, 673 (2003).

${ }^{10}$ S. R. Shinde et al., Phys. Rev. B 67, 115211 (2003).

${ }^{11}$ S. B. Ogale et al., Phys. Rev. Lett. 91, 077205 (2003).

${ }^{12}$ Y. G. Zhao et al., Appl. Phys. Lett. 83, 2199 (2003).

${ }^{13}$ Z. Wang, J. Tang, L. D. Tung, W. Zhou, and L. Spinu, J. Appl. Phys. 93, 7870 (2003)

${ }^{14}$ J. M. D. Coey, A. P. Douvalis, C. B. Fitzgerald, and M. Venkatesan, Appl. Phys. Lett. 84, 1332 (2004).

${ }^{15}$ N. H. Hong, J. Sakai, and A. Hassini, Appl. Phys. Lett. 84, 2602 (2004).

${ }^{16}$ J. He et al., Appl. Phys. Lett. 86, 052503 (2005).

${ }^{17}$ J. Philip et al., Nat. Mater. 5, 298 (2006).

${ }^{18}$ J. H. Cho et al., Appl. Phys. Lett. 88, 092505 (2006).

${ }^{19}$ A. Tiwari, V. M. Bhosale, S. Ramachandran, N. Sudhakar, J. Narayan, S. Budak, and A. Gupta, Appl. Phys. Lett. 88, 142511 (2006).

${ }^{20}$ K. Gopinadhan, D. K. Pandya, S. C. Kashyap, and S. Chaudhary, J. Appl. Phys. 99, 126106 (2006).

${ }^{21}$ N. H. Hong, N. Poirot, and J. Sakai, Appl. Phys. Lett. 89, 042503 (2006). 